\title{
Psychometric properties of a questionnaire evaluating knowledge and attitudes of dietetic professionals towards personalised nutrition and nutrigenomics
}

\author{
P. Moitra, J. Nemani and J. Madan \\ Department of Food, Nutrition \& Dietetics, Sir Vithaldas Thackersey College of Home Science (Autonomous), \\ SNDT Women's University, Mumbai, India
}

The growing interest in the potential of nutrigenomics and personalized nutrition to prevent, mitigate and manage chronic diseases necessitates a better understanding of nutrient-gene interactions among dietetic professionals. Information regarding the knowledge and attitudes of dietitians regarding nutrigenomics is, in general, limited ${ }^{(1)}$ and little is known about the perceived barriers, willingness to learn, and confidence to practice personalized nutrition (PN) among dietetic professionals in India. To our knowledge, there are no studies that have developed and evaluated the validity and reliability of questionnaires assessing the knowledge, attitudes, and behaviours related to nutrigenomics and personalized nutrition in India. Therefore, this study was conducted to test the psychometric properties of a novel instrument measuring knowledge and attitudes of dietetic professionals towards personalized nutrition and nutrigenomics in India.

Extensive review of the literature followed by five semi-structured focus group discussions with thirteen registered dietitians were conducted to generate an item pool. The face validity was evaluated among a sample of dietetic professionals $(n=18)$. The item content validity index (I-CVI) and scale CVI for clarity and relevance of items were assessed based on the responses of experts $(\mathrm{n}=5)$ with professional degrees in nutrigenomics and/ or $>5$ years of experience in the field of personalized nutrition counselling. To determine the item difficulty index (IDI), item discrimination index (DI), and internal consistency of the instrument, a cross-sectional sample of practicing dietitians $(n=44)$ completed the questionnaire. The confirmatory factor analysis method was used to determine the factor loadings, fit indices of Comparative Fit Index (CFI), Tucker Lewis Fit Index (TLI), and Root Mean Square Error of Approximation (RMSEA) for establishing the construct validity. The intraclass correlation coefficient values were calculated to determine test-retest reliability.

The mean age of the participants was 34.4 (12.4) years. The item difficulty indices ranged from 0.60 to 0.72 and the mean Cronbach alpha coefficient value was 0.82 indicating acceptable to excellent internal consistency of the questionnaire. The results of CFA presented a four-factor model with satisfactory goodness of fit indices- 1) level of knowledge of nutrigenomics, 2) attitude and willingness to adopt personalized nutrition, 3) behaviours related to applications of nutrigenomic in disease management, and 4) training and confidence to practice PN. The intraclass correlation coefficients for knowledge, attitude, behaviour, and training domains were $0.74,0.83,0.72$, and 0.81 , respectively.

Optimal results pertaining to the validity and reliability of the instrument were obtained. This novel questionnaire may prove useful to understand the knowledge and attitudes to nutrigenomics in dietetic professionals in India and can provide a basis to develop appropriate capacity building and training programs required to translate knowledge into clinical practice.

\section{References}

1. Kaufman-Shriqui V, Salem H, Boaz M, et al. (2020) Nutrients Feb; 12(2), 335. 